\title{
Primary hydatid cyst of head of pancreas communicating with common bile duct containing stones
}

\author{
D. Thakur, U. Somashekar, R. Kothari,V. Kumar, D. Sharma \\ Department of Surgery, Government NSCB Medical College, Jabalpur (MP), India.
}

Keywords: Echinococcosis; pancreas; obstructive jaundice

\section{Introduction}

Primary pancreatic involvement by Echinococcus granulosis is extremely rare [1]. Hydatid cyst of pancreatic head containing bile and stones with a biliary communication has never been reported.

\section{Case detail}

A 28 year female presented with dull pain in the upper abdomen for 1 year and distension/ fullness in abdomen and itching all over the body for 1 month. Examination showed icterus and a palpable gall bladder. Serum bilirubin was $4 \mathrm{mg} / \mathrm{DL}$, serum alkaline phosphatase was $700 \mathrm{IU} / \mathrm{L}$, Alanine transaminase was $103 \mathrm{IU} / \mathrm{L}$ and Aspartate transaminase was 201 IU/L. Magnetic Resonance Cholangio-Pancreaticography (MRCP) showed a thick walled cystic lesion with some solid component, measuring $8 \times 7 \times 6.1 \mathrm{~cm}$ at lower end of Common Bile Duct, with internal membranes and calcifications (Figure 1). Common Bile Duct (23mm), Common Hepatic Duct (13mm) and Intra Hepatic Biliary Radicals were dilated. Gall bladder showed multiple calculi. A provisional diagnosis of Hydatid cyst of head of pancreas was made. Hydatic serology was not done due to its non-availability. Patient was given 400 $\mathrm{mg}$ of Albendazole, once a day for 3 weeks and then was taken up for surgery.

On exploration a large cystic mass was found in the head of pancreas, which was not fixed to surrounding structures. Intra-operative fine needle aspiration showed frank bile, causing suspicion of choledochal cyst. Pancreatico-duodenal resection was done (Figure 2 ). Resected specimen was cut open after the procedure

Correspondence: D. Sharma

E-mail: dhanshar@gmail.com and revealed a cystic lesion containing hydatid cyst (Figure 3). It also showed a large biliary communication

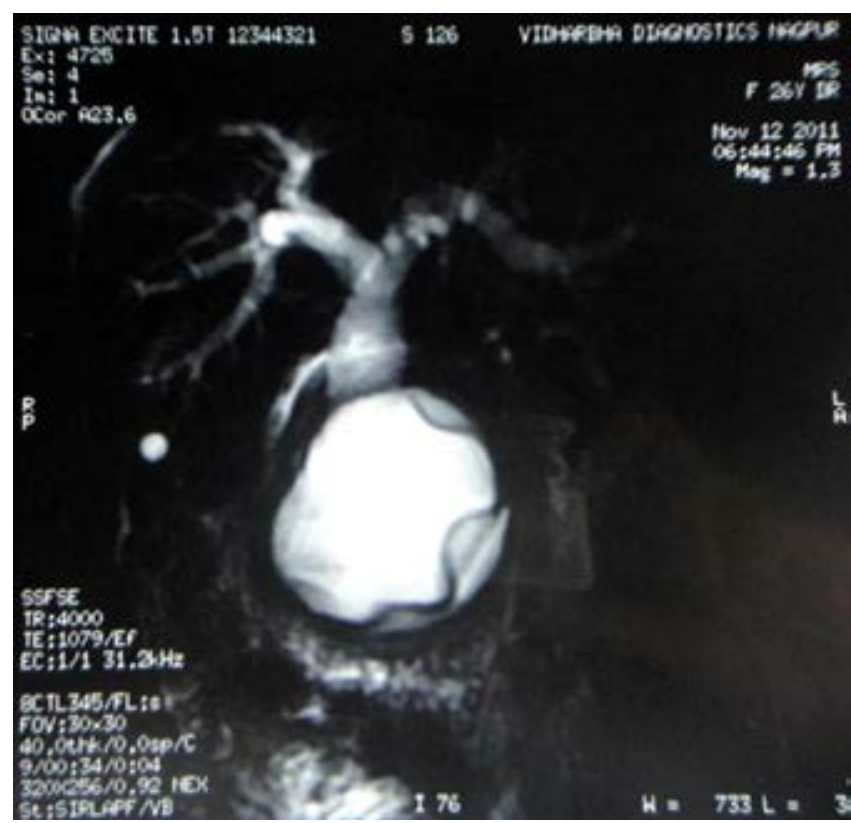

Figure 1. MRCP showing thick walled cystic lesion with some solid component, measuring $8 \times 7 \times 6.1 \mathrm{~cm}$ at lower end of CBD Duct, with internal membranes and calcifications; with dilated CBD and Intra Hepatic Biliary Radicals

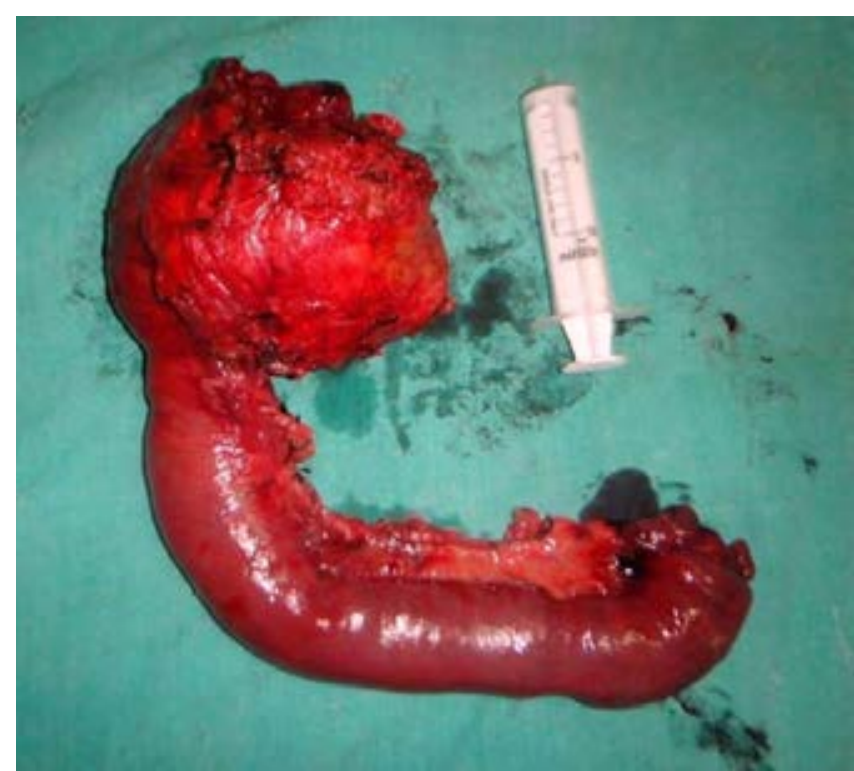

Figure 2. Pancreatico-duodenal resection specimen showing cystic mass in the head region 
with a few stones and bile contained within the cyst (Figure 4). Histopathological examination confirmed the diagnosis. Post-operative recovery was uneventful.

\section{Discussion}

Pancreatic hydatid disease is quite rare. Logically, hydatid disease should be considered in the differential diagnosis of all cystic masses in the pancreas, even if Casoni and indirect hemagglutination tests are negative, especially in geographic regions where the disease is endemic. Familiarity with the imaging findings of hydatid disease is helpful in suspecting the diagnosis and thereby preventing potential complications [2]. Standard treatment is resection for cysts in body or tail, while a cystectomy with drainage or pancreaticoduodenal resection is needed for lesions in the head of pancreas $[3,4]$.

A review of literature failed to reveal a previous publication showing pancreatic head hydatid cyst

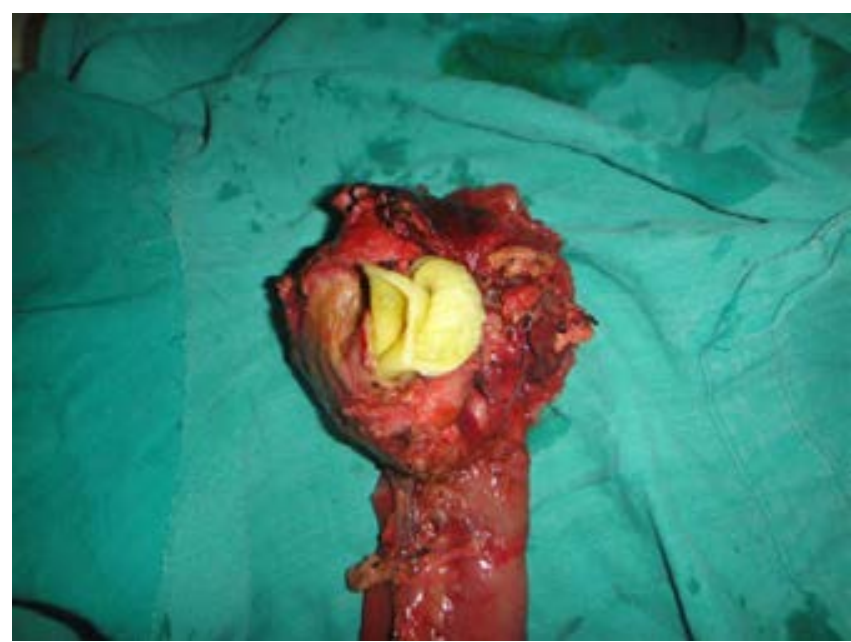

Figure 3. Resected specimen cut open to show hydatid cyst.

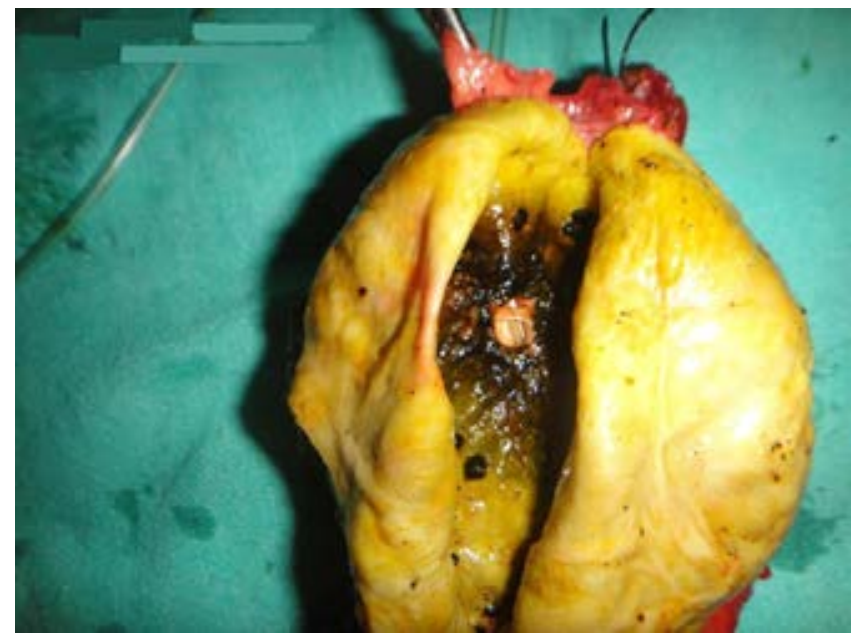

Figure 4. Hydatid cyst cut open showing a large biliary communication with a few stones within the cyst.

containing bile and stones with a biliary communication.

\section{References}

1. Hamamci EO, Besim H, Korkmaz A. Unusual locations of hydatid disease and surgical approach. ANZ J Surg. 2004 May;74(5):356-60.

2. Yuksel M, Demirpolat G, Sever A, Bakaris S, Bulbuloglu E, Elmas N. Hydatid disease involving some rare locations in the body: a pictorial essay. Korean J Radiol. 2007 NovDec;8(6):531-40.

3. Shah OJ, Robbani I, Zargar SA, Yattoo GN, Shah P, Ali S, et al. Hydatid cyst of the pancreas. An experience with six cases. JOP. 2010;11(6):575-81

4. Khiari A, Mzali R, Ouali M, Kharrat M, Kechaou MS, Beyrouti MI. Hydatid cyst of the pancreas. Apropos of 7 cases. Ann Gastroenterol Hepatol (Paris). 1994 MayJun;30(3):87-91. [Article in French]

\section{Key points:}

- Hydatid disease should be considered in all cystic masses of the pancreas, although rare. This is essential in areas where the disease has a high prevalence. 